\title{
Determination of Optimal Number of Servers at Network Queuing Nodes to Reduce Waiting Time in a Tertiary Institution Clinic in Bida, Nigeria
}

\author{
"MUHAMMAD, I; ADAMU, L \\ Department of Mathematics, Federal University of Technology Minna, Nigeria \\ *Corresponding Author Email: lawal.adamu@futminna.edu.ng
}

\begin{abstract}
In this paper, a network queuing model that determines optimal numbers of servers at the nodes of the school clinic network queuing system to reduce waiting time of the patients has been presented. The relevant data was collected for a period four weeks, through direct observations and interviews. The number of arrivals and departures were also obtained. The total expected waiting time of the patient in the current system before modification was 50minutes with total number of 10 servers in all the nodes, while the total new expected waiting time of patient in the system after modification was reduced to 19 minutes with total number of 17 servers in all the nodes. The study has determined optimal number of servers at the nodes of the school clinic network system. Results from this study is an important information to the management of the school clinic for proper planning and better service delivery.
\end{abstract}

\section{DOI: https://dx.doi.org/10.4314/jasem.v24i9.22}

Copyright: Copyright (C) 2020 Muhammad and Adamu. This is an open access article distributed under the Creative Commons Attribution License (CCL), which permits unrestricted use, distribution, and reproduction in any medium, provided the original work is properly cited.

Dates: Received: 10 August 2020; Revised: 16 September 2020; Accepted: 22 September 2020

Keywords: Network Queuing System, Nodes, Servers, School Clinic.

In Nigeria, It is a common knowledge that patients spent a lot of their times on queues waiting to be treated (Lawal et al., 2019).Waiting for service in real life, is a common phenomenon in Nigeria and in around the world. We wait for services in hospitals, gas stations and bus stops, we queue up for service in banks, schools, general stores, and so on, service delay is unavoidable as a system gets blocked (Kandenmir and Cavas, 2007). The Federal Polytechnic Bida school clinic is not left out from this avoidable time wasting. The majority of patients receiving treatments in Federal Polytechnic Bida school clinic are students and staff; this protracted waiting time on queue had often affected the academic performance of the students as a result of missing lectures by both the lecturers and students. These times wasting is mostly as a result of shortage of servers in some departments of the clinic or too many servers in some departments where they are not needed this has created redundancies and deficiencies in some departments of the clinic. Queuing theory is considered to be branch of operations research. It constitutes a powerful tool in modeling and performance analysis of many complex systems, such as computer networks, hospital network system, telecommunication systems, call centre, flexible manufacturing systems and service systems. Recently, the queuing theory including queuing systems and networks arouse mathematicians', engineers' and economics interests. It is clear that the queuing system in the school clinic is a network type because mostly patients move from one service facilities to another within the clinic in order to complete their treatments. Network of queues are used to model possible conflict of queuing when a set of resources are shared. It is a model in which jobs departing from one queue arrive at another, it describes a situation where the input from one queue is the output of one or more queues. Example of where queuing network can be applied are machine shops, communication network, hospital system, and movement of memos within an organization just to mention a few. The following are some of the researches found in the literature on queuing systems. Danish Engineer, A.K Erlang in 1913 first analyzed queue or queuing theory or waiting lines in the context of telephone facilities. He started with the problem of the congestion of telephone traffic and later on, extended to business application and waiting lines. The ideas have since seen applications in telecommunication, traffic engineering, computing and the design of factories, shops, offices and hospitals (Schelechter, 2009).Lawal et al., (2019) have studied the waiting 
and service costs of a multi-server queuing system at National Health Insurance scheme (NHIS) unit of General Hospital Minna, Niger state, Nigeria. The two conflicting costs were balanced and the optimal performance for the queuing system was then determined. It was concluded that for the morning session, the average queuing length, waiting time of patients as well as overutilization of doctors at the unit could be reduced at an optimal sever level of 3 doctors at a minimum total cost of $\$ 6219.98$ per hour as against the present server level of 2 doctors with high total cost of $\$ 26025.12$ per hour which include waiting and service costs and for the evening session the present sever level of 1 doctor should be maintained. The researchers recommend that the result of the research is an important information to the management of NHIS unit of the General Hospital Minna to provide better service to the patients at a minimum cost. A successful application of queuing system in hospital management has also been reported in (John, 2010; Olaniyi, 2004; Kebe and Onah, (2012); Lakshmi, and Sivakumar, 2013; Adaji, 2018). Also, researchers such as (Damondhar and shastraka, 2018), (Muniratet al., 2015), (Nityangini and Pravin, 2016), (Shastrakar and Pokley, 2017) and (Sushilet al., 2017) had successfully applied queuing theory in other fields.

\section{MATERIALS AND METHODS}

The type of queuing system adopted by an organizations solely dependent on the type of service being provided. The Federal Polytechnic school clinic practice network type of queuing system. Queuing network is composed of several random queue systems, mostly limited and single queue systems. Diverse types of patients go by through the network in many ways and are served by the service nodes within the network system. A queuing network system has a set of nodes $(i)$. Each node has a number of servers $(s)$ and a single node can be regarded as a queuing system. Patients can have access to the queuing network from any node. The arrival rate from the outside is $\lambda$ and the arrival rate of Node $i$ is $\lambda_{i}$. After the patients queues and gets the service at a node (the service rate of Node $i$ is $\mu_{i}$ ), he/she can leave the network system or go to another node, or even return to the former node.

Model Formulation: The Federal Polytechnic Bida is a Federal owned tertiary institution, located in Bida Local Government of Niger state, Nigeria. The school clinic is within the campus and is made up of six different departments. In this study, each department is regarded as node of the network system. The data used in this research were gathered from the six departments of the Federal Polytechnic Bida school clinic and they were obtained based on the arrival and departure rate as well time spent at each node. The method of data gathering was direct observation and personal interview by the research team. The data gathering was for a period of four weeks. This data gathering was carried from Monday to Sunday. In a day, gathering of the data was for a total of six (6) hours at different times of the day. For each node, the number of arrivals and departures together with service times were taken at intervals of 4 minutes arrivals of patients into a node $(\lambda)$, while the departure rate was obtained also by the average number of four (4) minutes departures of patients $(\mu)$ at that particular node. Each of the nodes was observed for a period of one (1) hour daily. All the nodes under consideration have at least a server.

Model Assumptions: We make the following assumptions for Federal Polytechnic School clinic.

1. The Federal Polytechnic Bida school clinic network queuing system is considered

as an independent queuing system

2. Queuing discipline in Federal Polytechnic Bida school clinic is first come first served.

3. The external arrival pattern follow a Poisson arrival process.

4. Each node in the Federal Polytechnic school clinic has at least a server with exponential services time.

5. The service rate in the school clinic depends on the number of patients at each node of the system

6. There is no limited capacity in the Federal Polytechnic school clinic for the arriving

Patients and each service facility has unlimited waiting space.

7. In the school clinic the medical personnel's are regarded as servers

8. All the medical service provider in the school clinic are working in full capacity

9. Service rate is independent of queuing length.

The study of the school clinic queuing system is based on Jackson open network model, the Federal polytechnic school clinic is made up of the following departments, the Registration, nursing, Consultation, Pharmacy, Laboratory and Accounting. We assumed in this research that patients who come into the Federal Polytechnic school clinic for services will commence by going first to the registration unit to register and then proceed to the nursing unit, from 
there, patients move to see the doctor at the consultation unit, this procedure continue until the patients depart from the clinic. At each department in school clinic, there is only one queue and one service at a time.

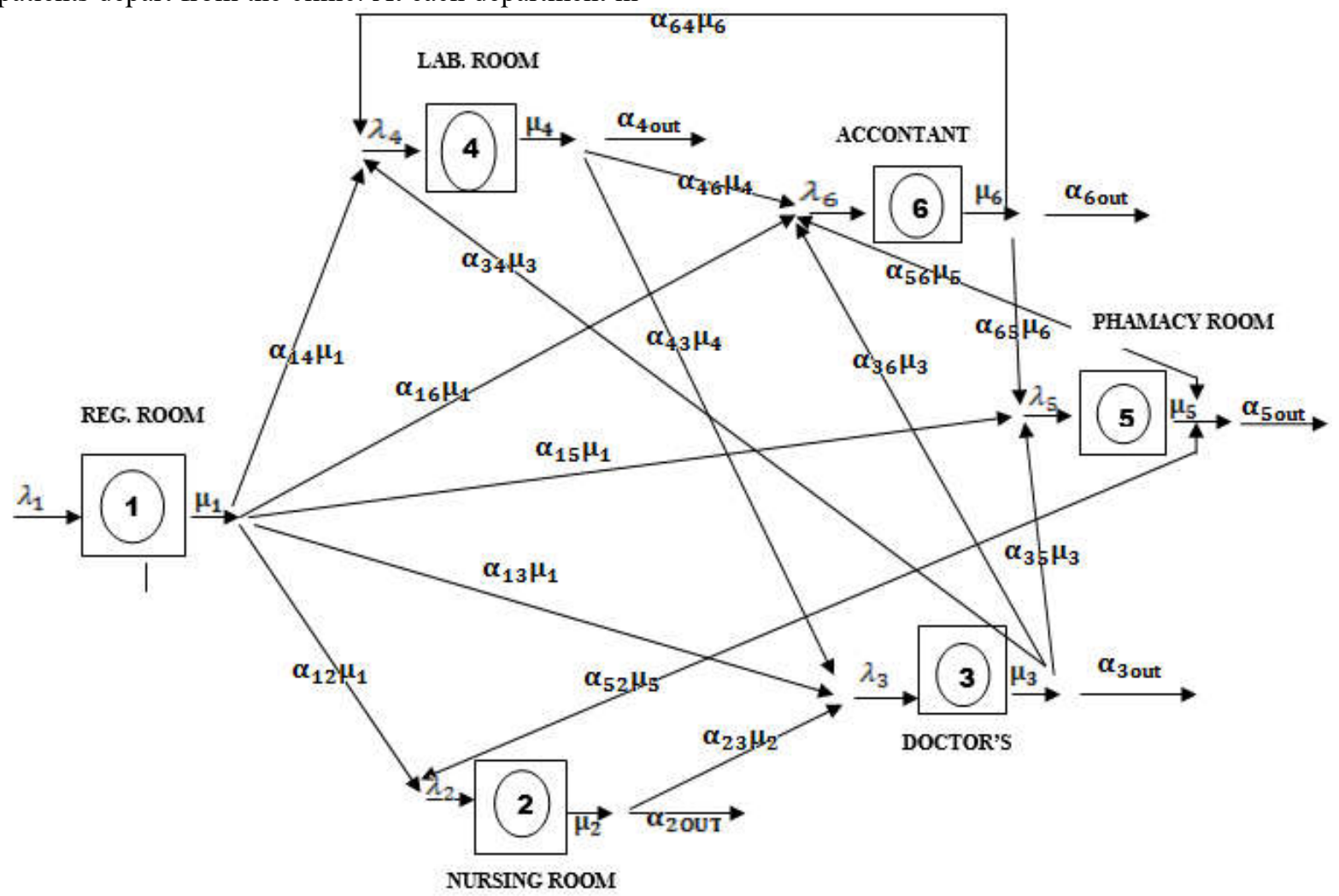

Fig 1.Schematic diagram of Federal Polytechnic school clinic network queuing system.

Where: $\lambda_{i}$ is the arrival rate of the patients, for $i=1,2$, .6; $\boldsymbol{\mu}_{i}$ is the departure rate of the patients, for $\mathrm{i}=1,2$, $.6 ; \alpha_{i j}$ are the weight of moving from nodei to node $\mathrm{j}$; $m_{i}$ is the number of servers at the various node in the network system; for $i=1,2, \quad 6$.

The notations used for the presentation of the data in the school clinic are node 1 , node 2 , node 3 , node 4 , node 5 and node 6 , all through the period of 4 weeks, which includes the number of arrivals and number of departures with an interval of 4 minutes.

They are denoted as follow: Registration point denoted by node 1 ; Nursing station point denoted by node 2; Doctors' Room is denoted by node 3; Laboratory unit is denoted by node 4; Pharmacy unit denoted by node 5 ; Accountant's denoted by node 6

The arrival rate

$$
\lambda_{i}=\frac{1}{\text { mean number of amival }}
$$

for $i=2 \ldots .6$.
The departure rate

$$
\begin{aligned}
& \mu_{i}=\frac{1}{\text { mean number of departure }}, \quad \text { for } \mathrm{i}= \\
& 1,2, \ldots \ldots .6 \quad \text { (2) } \\
& \rho=\frac{\lambda_{\mathrm{i}}}{\mu_{\mathrm{i}}}, \text { for } i=1,2, \ldots \ldots 6
\end{aligned}
$$

The expected number of patients on the queue is given as

$l_{q}=\frac{\rho}{m-\rho}$

Where $\mathrm{m}$ stands for the number of servers at the node The expected waiting time of the patients on the queue is given as:

$w_{q i}=\frac{l_{\pi}}{\lambda_{i}{ }^{\prime}}$

The expected number of in the system is given as

$l_{z}=l_{q}+\rho$

The expected waiting time of the patients in the system for node 1-6 is given as

$w_{i}=\frac{l_{s}}{\lambda_{i}}$, for $\mathrm{i}=1,2, \ldots \ldots \ldots \ldots$.

Model Equations 
From Figure1, we obtained the following model equations

$\lambda_{2}=\alpha_{12} \mu_{1}+\alpha_{52} \mu_{5}$

$\lambda_{3}=\alpha_{13} \mu_{1}+\alpha_{23} \mu_{2}+\alpha_{43} \mu_{4}$

$\lambda_{4}=\alpha_{14} \mu_{1}+\alpha_{34} \mu_{3}+\alpha_{64} \mu_{6}$

$\lambda_{5}=\alpha_{15} \mu_{1}+\alpha_{35} \mu_{3}+\alpha_{65} \mu_{6}$

$\lambda_{6}=\alpha_{16} \mu_{1}+\alpha_{36} \mu_{3}+\alpha_{46} \mu_{4}+\alpha_{56} \mu_{5}$

Also,

$$
\begin{aligned}
& \mu_{1}=\alpha_{12} \mu_{1}+\alpha_{13} \mu_{1}+\alpha_{14} \mu_{2}+\alpha_{15} \mu_{1}+\alpha_{16} \mu_{5} \\
& \mu_{2}=\alpha_{23} \mu_{2}+\alpha_{2 o u t} \mu_{2} \\
& \mu_{3}=\alpha_{34} \mu_{3}+\alpha_{35} \mu_{3}+\alpha_{36} \mu_{6}+\alpha_{3 o u t} \mu_{3} \\
& \mu_{4}=\alpha_{43} \mu_{4}+\alpha_{46} \mu_{4}+\alpha_{4 o u t} \mu_{4} \\
& \mu_{5}=\alpha_{52} \mu_{5}+\alpha_{56} \mu_{5}+\alpha_{5 o u t} \mu_{5} \\
& \mu_{6}=\alpha_{64} \mu_{6}+\alpha_{65} \mu_{6}+\alpha_{6 o u t} \mu_{6}
\end{aligned}
$$

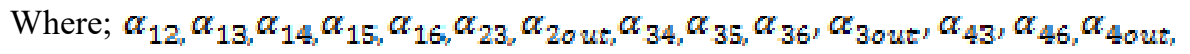

$\alpha_{52}, \alpha_{56}, \alpha_{5 o u t} \alpha_{64}, \alpha_{60 u t}$ are to determined

Model equations (8-18) could be expressed as:

$$
\begin{aligned}
& \lambda_{2}=\mu_{1} \alpha_{12}+0 \alpha_{13}+0 \alpha_{14}+0 \alpha_{15}+0 \alpha_{16}+0 \alpha_{23}+0 \alpha_{20 u t}+0 \alpha_{34}+0 \alpha_{35} \\
& +0 \alpha_{36}+0 \alpha_{3 o u t}+0 \alpha_{43}+0 \alpha_{46}+0 \alpha_{40 u t}+\mu_{5} \alpha_{52}+0 \alpha_{56}+0 \alpha_{\text {sout }} \\
& +0 \alpha_{64}+0 \alpha_{65}+0 \alpha_{60 u t} \\
& \lambda_{3}=0 \alpha_{12}+\mu_{1} \alpha_{13}+0 \alpha_{14}+0 \alpha_{15}+0 \alpha_{16}+\mu_{2} \alpha_{23}+0 \alpha_{202 t}+0 \alpha_{34}+0 \alpha_{35} \\
& +0 \alpha_{36}+0 \alpha_{3 o u t}+\mu_{4} \alpha_{43}+0 \alpha_{46}+0 \alpha_{40 u t}+0 \alpha_{52}+0 \alpha_{56}+0 \alpha_{\text {5out }} \\
& 0 \alpha_{64}+0 \alpha_{65}+0 \alpha_{6 o u t} \\
& \lambda_{4}=0 \alpha_{12}+0 \alpha_{13}+\mu_{1} \alpha_{14}+0 \alpha_{15}+0 \alpha_{16}+0 \alpha_{23}+0 \alpha_{2 o u t}+\mu_{3} \alpha_{34}+0 \alpha_{35} \\
& +0 \alpha_{36}+0 \alpha_{3 o u t}+0 \alpha_{43}+0 \alpha_{46}+0 \alpha_{4 o u t}+0 \alpha_{52}+0 \alpha_{56}+0 \alpha_{5 \sigma u t} \\
& +0 \alpha_{56}+0 \alpha_{\text {soust }}+\mu_{6} \alpha_{64}+0 \alpha_{65}+0 \alpha_{6 o u t} \\
& \lambda_{5}=0 \alpha_{12}+0 \alpha_{13}+0 \alpha_{14}+\mu_{1} \alpha_{15}+0 \alpha_{16}+0 \alpha_{23}+0 \alpha_{2 o u t}+0 \alpha_{34}+\mu_{3} \alpha_{35} \\
& +0 \alpha_{36}+0 \alpha_{30 u t}+0 \alpha_{43}+0 \alpha_{46}+0 \alpha_{40 u t}+0 \alpha_{52}+\mu_{5} \alpha_{56}+0 \alpha_{\text {sout }} \\
& +0 \alpha_{64}+\mu_{6} \alpha_{65}+0 \alpha_{60 u t} \\
& \lambda_{6}=0 \alpha_{12}+0 \alpha_{13}+0 \alpha_{14}+0 \alpha_{15}+\mu_{1} \alpha_{16}+0 \alpha_{23}+0 \alpha_{20 u t}+0 \alpha_{34}+0 \alpha_{35} \\
& +\mu_{3} \alpha_{36}+0 \alpha_{30 u t}+0 \alpha_{43}+\mu_{4} \alpha_{46}+0 \alpha_{40 u t}+0 \alpha_{52}+\mu_{5} \alpha_{56}+0 \alpha_{\text {5out }} \\
& +0 \alpha_{64}++0 \alpha_{65}+0 \alpha_{6 o u t} \\
& \text { Also, } \\
& \mu_{1}=\mu_{1} \alpha_{12}+\mu_{1} \alpha_{13}+\mu_{1} \alpha_{14}+\mu_{1} \alpha_{15}+\mu_{1} \alpha_{16}+0 \alpha_{23}+0 \alpha_{2 o u t}+0 \alpha_{34}+0 \alpha_{35} \\
& +0 \alpha_{36}+0 \alpha_{3 o u t}+0 \alpha_{43}+0 \alpha_{46}+0 \alpha_{40 u t}+0 \alpha_{52}+0 \alpha_{56}+0 \alpha_{5 o u t} \\
& +0 \alpha_{64}+0 \alpha_{65}+0 \alpha_{\text {Sout }} \\
& \mu_{2}=0 \alpha_{12}+0 \alpha_{13}+0 \alpha_{14}+\mu_{1} \alpha_{15}+0 \alpha_{16}+\mu_{2} \alpha_{23}+\mu_{2} \alpha_{20 u t}+0 \alpha_{34}+0 \alpha_{35} \\
& +0 \alpha_{36}+0 \alpha_{3 o u t}+0 \alpha_{43}+0 \alpha_{46}+0 \alpha_{4 o u t}+0 \alpha_{52}+\mu_{5} \alpha_{56}+0 \alpha_{5 o u t} \\
& +0 \alpha_{64}+0 \alpha_{65}+0 \alpha_{60 u t} \\
& \mu_{3}=0 \alpha_{12}+0 \alpha_{13}+0 \alpha_{14}+\mu_{1} \alpha_{15}+0 \alpha_{16}+0 \alpha_{23}+0 \alpha_{20 u t}+\mu_{3} \alpha_{34}+\mu_{3} \alpha_{35} \\
& +\mu_{3} \alpha_{36}+\mu_{3} \alpha_{3 \text { out }}+0 \alpha_{43}+0 \alpha_{46}+0 \alpha_{4 o u t}+0 \alpha_{52}+0 \alpha_{56}+0 \alpha_{5 o u t} \\
& +0 \alpha_{64}+0 \alpha_{65}+0 \alpha_{6 o u t} \\
& \mu_{4}=0 \alpha_{12}+0 \alpha_{13}+0 \alpha_{14}+\mu_{1} \alpha_{15}+0 \alpha_{16}+0 \alpha_{23}+0 \alpha_{20 u t}+0 \alpha_{34}+0 \alpha_{35} \\
& +0 \alpha_{36}+0 \alpha_{3 o u t}+\mu_{4} \alpha_{43}+\mu_{4} \alpha_{46}+\mu_{4} \alpha_{40 u t}+0 \alpha_{52}+0 \alpha_{56}+0 \alpha_{5 o u t}
\end{aligned}
$$




$$
\begin{aligned}
& +0 \alpha_{64}+0 \alpha_{65}+0 \alpha_{6 o u t} \\
& \mu_{5}=0 \alpha_{12}+0 \alpha_{13}+0 \alpha_{14}+\mu_{1} \alpha_{15}+0 \alpha_{16}+0 \alpha_{23}+0 \alpha_{2 o u t}+0 \alpha_{34}+0 \alpha_{35} \\
& +0 \alpha_{36}+0 \alpha_{3 o u t}+0 \alpha_{43}+0 \alpha_{46}+0 \alpha_{4 o u t}+\mu_{5} \alpha_{52}+\mu_{5} \alpha_{56}+\mu_{5} \alpha_{5 o u t} \\
& +0 \alpha_{64}+0 \alpha_{65}+0 \alpha_{6 o u t} \\
& \mu_{6}=0 \alpha_{12}+0 \alpha_{13}+0 \alpha_{14}+0 \alpha_{15}+0 \alpha_{16}+0 \alpha_{23}+0 \alpha_{2 o u t}+0 \alpha_{34}+0 \alpha_{35} \\
& +0 \alpha_{36}+0 \alpha_{3 o u t}+0 \alpha_{43}+0 \alpha_{46}+0 \alpha_{4 o u t}+0 \alpha_{52}+0 \alpha_{56}+0 \alpha_{5 o u t} \\
& +\mu_{6} \alpha_{64}+\mu_{6} \alpha_{65}+\mu_{6} \alpha_{6 o u t}
\end{aligned}
$$

Model equations (19-29) above can be represented in the matrix form as:

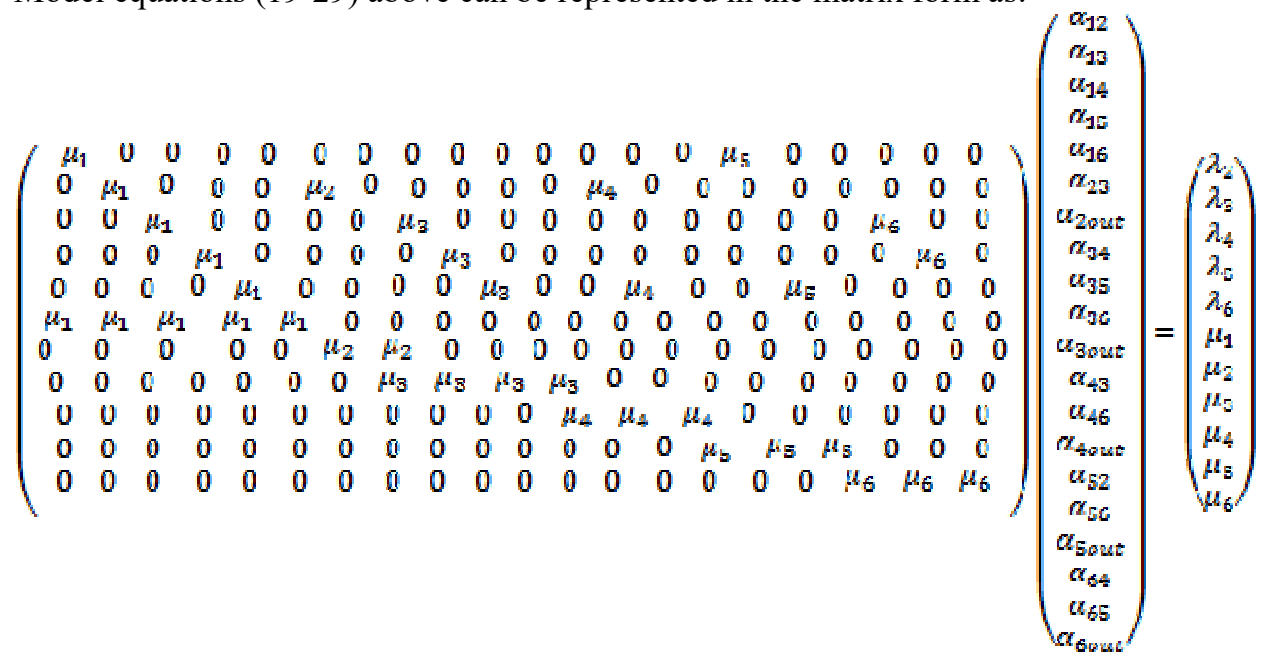

Mathematical Formulation of New Departure Rate:

For us to be able to minimize the waiting time, which is the main aim of this research work, there is need to introduce a new departure rate for each node.

Therefore, model equations (8-12) will generate equations (31 - 35) below;

$\lambda_{2}=\alpha_{12} \mu_{1}+\alpha_{52} \mu_{5}$

$\lambda_{3}=\alpha_{13} \mu_{1}+\alpha_{23} \mu_{2}+\alpha_{43} \mu_{4}$

$\lambda_{4}=\alpha_{14} \mu_{1}+\alpha_{34} \mu_{3}+\alpha_{64} \mu_{6}$

$\lambda_{\mathrm{s}}=\alpha_{15} \mu_{1}+\alpha_{35} \mu_{3}+\alpha_{65} \mu_{6}$

$\lambda_{6}=\alpha_{16} \mu_{1}+\alpha_{36} \mu_{3}+\alpha_{46} \mu_{4}+\alpha_{56} \mu_{5}$

Where all $\alpha_{i}^{\prime} s$ and $\lambda_{i}^{\prime} s$ are know

Equation $(31-35)$ becomes

$\lambda_{2}=\alpha_{12} \mu_{1}+0 \mu_{2}+0 \mu_{3}+0 \mu_{4}+\alpha_{52} \mu_{5}+0 \mu_{6}$

$\lambda_{3}=\alpha_{13} \mu_{1}+\alpha_{23} \mu_{2}+0 \mu_{3}+\alpha_{43} \mu_{4}+0 \mu_{5}+0 \mu_{6}$

$$
\begin{aligned}
\lambda_{4} & =\alpha_{14} \mu_{1}+0 \mu_{2}+\alpha_{34} \mu_{3}+0 \mu_{4}+0 \mu_{5}+\alpha_{64} \mu_{6} \\
\lambda_{5} & =\alpha_{18} \mu_{1}+0 \mu_{2}+a_{38} \mu_{3}+0 \mu_{4}+0 \mu_{5}+\alpha_{66} \mu_{6} \\
\lambda_{4} & =\alpha_{16} \mu_{1}+0 \mu_{2}+a_{36} \mu_{3}+\alpha_{46} \mu_{4}+a_{56} \mu_{5}+0 \mu_{6}
\end{aligned}
$$

Equations (36 - 40) are represented by equation (41)

$$
\left(\begin{array}{cccccc}
\alpha_{12} & 0 & 0 & 0 & \alpha_{52} & 0 \\
\alpha_{13} & \alpha_{23} & 0 & \alpha_{43} & 0 & 0 \\
\alpha_{14} & 0 & \alpha_{34} & 0 & 0 & \alpha_{64} \\
\alpha_{15} & 0 & \alpha_{35} & 0 & 0 & \alpha_{65} \\
\alpha_{16} & 0 & \alpha_{36} & \alpha_{46} & \alpha_{56} & 0
\end{array}\right)\left(\begin{array}{l}
\mu_{1} \\
\mu_{2} \\
\mu_{3} \\
\mu_{4} \\
\mu_{5} \\
\mu_{6}
\end{array}\right)=\left(\begin{array}{l}
\lambda_{2} \\
\lambda_{3} \\
\lambda_{4} \\
\lambda_{5} \\
\lambda_{6}
\end{array}\right)
$$

\section{RESULTS AND DISCUSSION}

We begin this section with computation of mean arrival and departure time for all the nodes in the network system, the results of the computation is presented in Table1.

Table 1: The Mean arrival and Mean departure time obtained for each of the nodes from the raw data collected at the Clinic.

\begin{tabular}{lllllll}
\hline & Node 1 & Node 2 & Node 3 & Node 4 & Node 5 & Node 6 \\
\hline Mean arrival & 1.809 & 1 & 1.819 & 1.632 & 1.676 & 1.791 \\
Mean departure & 1.767 & 1 & 1.265 & 1.468 & 1.491 & 1.432 \\
\cline { 2 - 7 }
\end{tabular}


To obtain solution for equation (30), we use the mean arrival and mean departure time obtained from the raw data collected as presented in Table 1 above to calculate the arrival rate and departure rate using equation (1) and (2) for

$$
\left.\lambda_{i}, i=2, \ldots, 6 \text { and } \mu_{i}, i=1, \ldots, 6\right) \text { then }
$$

substitute them into equation (30). Hence, we obtained the solution for equation (30) as presented in equation (47) below

$$
\left(\begin{array}{c}
\alpha_{12} \\
\alpha_{13} \\
\alpha_{14} \\
\alpha_{15} \\
\alpha_{16} \\
\alpha_{23} \\
\alpha_{2 o u t} \\
\alpha_{34} \\
\alpha_{35} \\
\alpha_{36} \\
\alpha_{\text {sout }} \\
\alpha_{43} \\
\alpha_{46} \\
\alpha_{4 o u t} \\
\alpha_{\text {B2 }} \\
\alpha_{\text {b6 }} \\
\alpha_{5 o u t} \\
\alpha_{64} \\
\alpha_{65} \\
\alpha_{6 o u t}
\end{array}\right)-\left(\begin{array}{l}
0.6313043478 \\
0.0678260896 \\
0.1234782609 \\
0.12000000000 \\
0.05739130435 \\
0.42800000000 \\
0.57200000000 \\
0.3256743257 \\
0.3196803197 \\
219780219800 \\
0.1348651349 \\
0.2437562438 \\
0.4145854146 \\
0.3416503417 \\
0.8741258741 \\
0.0989010989 \\
0.02697302697 \\
0.38971315530 \\
0.3847675569 \\
0.2255192878
\end{array}\right)
$$

From equation (47), based on the result, the following deductions are made:

At node 1(Registration unit), the weights

$\alpha_{12}, \alpha_{13}, \alpha_{14}, \alpha_{15}$ and $\alpha_{16}$ are 0.6313043478 , $0.0678260896,0.1234782609$, 0.12000000000 , and 0.05739130435 respectively shows that there is a high probability of a patient leaving node 1 (registration) to join the queue for service at node 2 (Nurse unit) than any other node. The least probability is that a patient leaves node 1 to node 6 .

At node2 (Nurses unit), the weights $\alpha_{23}$ and $\alpha_{2 \text { out }}$ which are 0.42800000000 and 0.57200000000 which shows that there is a high probability that a patient leaves node 2 and goes directly to node 3 (Doctor Unit).

At node3 (Doctors point), the weights $\alpha_{34}, \alpha_{35}, \alpha_{36}$ and $\alpha_{3001}$ which 0.3256743257 0.3196803197 , $219780219800,0.1348651349$ respectively shows that there is a high probability that a patient leaves node 3 (Doctor unit) to join the queue for service either at node 4 or node 5 . The least probability is that a patient leaves node 3 (Doctor unit) and moves out of the system.
At node 4 (Laboratory point), the weights $\alpha_{43}, \alpha_{46_{u}}$ and $\alpha_{4 \text { sut }}$ which are 0.2437562438 , $0.4145854146, \quad$ and 0.3416583417 respectively shows that of 2 weight, there is a higher probability that a patient leaves node 4 and go back to node 3 (i.e to see Doctor) than out of the system.

At node5 (Pharmacy point), the weights $n_{52}, \alpha_{56}$, and $\alpha_{5 w u}$ which are 0.8741258741 , 0.0989010989 and 0.02697302697 respectively shows that there is a high probability that a patients leaves node 5 to join the queue for service either at node 2 or node 6 . The least probability here is that a patient leaves node 5 and goes out of the system.

At node6 (Account point), the weights $\alpha_{64}, \alpha_{65}$ and $\alpha_{60 u t}$ which are $0.38971315530, \quad 0.3847675569$ and 0.2255192878 respectively shows that there is a high probability that a patient leaves node 6 (Account unit) to join the queue for service either at node 4 or node 5. The least probability here is that a patient's leaves node 6 and goes out of the system

From the information in above Table 1, we have calculated the arrival and departure rates for each node, expected number of patient in the system, expected number of patient in the queue, expected waiting time in the system and expected waiting time in the queue.

For node 1, the arrival

rate $\lambda_{1}=\frac{1}{\text { meannamberofarrival }}=\frac{1}{1.809}=0.55$

person per minute.

The departure rate for node 1 is given as:

$\mu_{1}=\frac{1}{\text { manwamberofdepartwere }}=\frac{1}{1.767}=0.566$

Hence, we followed the same procedure to calculate for node 2 - to node 6 , the values obtained are presented in Table 2. Other values of the performance measure for all the nodes are computed and presented in Table 2.

$\rho=\frac{\lambda_{1}}{\mu_{1}}=\frac{0.55}{0.566}=0.97$

The expected number of patients in the queue is given as

$l_{q}=\frac{\rho}{m-\rho}=\frac{0.97}{4-0.97}=0.32$ Patient. 
Where $\mathrm{m}$ stands for the number of servers at the node1 (Registration point).

The expected waiting time of the patients on the queue is given as $w_{q 1}=\frac{l_{q}}{\lambda_{1}}=\frac{32}{0.55}=0.59$ minute

The expected number of patients in the system is given as

$l_{s}=l_{q}+\rho=0.32+0.97=1.29 \approx 1.3 \mathrm{P}$ atients

The expected waiting time in the system for node 1 is given as $w_{1}=\frac{\sharp_{s}}{\lambda_{1}}=\frac{1.3}{0.55}=2.5$ minutes. These parameters calculated for node 1 can be done for nodes $2-6$.
The expected waiting time in node 1 through node 6 were calculated as $\mathrm{w}_{1}=5$ minutes for node $1, \mathrm{w}_{2}=2$ minutes for node $2, \mathrm{w}_{3}=5.5$ minutes, for node $3, \mathrm{w}_{4}$ $=16.4$ minutes for node $4, \mathrm{w}_{5}=15$ minutes for node 5 and $\mathrm{w} 6=8.9$ minutes for node 6 . Therefore, the total expected waiting time of the patients in the current system is computed as

$$
\begin{aligned}
& W=w_{1}+w_{2}+w_{3}+w_{4}+w_{5}+w_{6}= \\
& 5+2+5.5+16.4+15+8.9=
\end{aligned}
$$

\section{$50.3 \approx 50$ minutes.}

The values of these performance measures of the network system are presented in the Table 2 below.

\begin{tabular}{lllllll}
\multicolumn{6}{c}{ Table 2: Performance measure for the current network system at the clinic } \\
\hline Nodes i & $\begin{array}{l}\text { Number } \\
\text { of Servers }\end{array}$ & $\rho_{\mathrm{i}}$ & $\mathrm{Lq}$ & $\mathrm{Ls}$ & $\mathrm{W}_{\mathrm{q}}$ & $\mathrm{W}_{\mathrm{S}}$ \\
\hline 1 & 4 & 0.97 & 0.3 & 1.3 & 0.59 & 2.5 \\
2 & 2 & 1.00 & 1.0 & 2.0 & 1.00 & 2.0 \\
3 & 1 & 0.70 & 2.3 & 3.0 & 4.18 & 5.5 \\
4 & 1 & 0.90 & 9.0 & 10 & 14.75 & 16.4 \\
5 & 1 & 0.89 & 8.0 & 9.0 & 13.30 & 15 \\
6 & 1 & 0.80 & 4.0 & 5.0 & 7.14 & 8.9 \\
Total & 10 & 5.26 & 24.6 & 30.3 & 40.96 & 50.3 \\
\hline
\end{tabular}

Solution for New Departure Rate: To obtain the new departure rate for the network system, we solve equation (41), this is done by substituting the values of arrival rate obtained in the previous section into equation (41) then we obtain equation (43)

$$
\left(\begin{array}{cccccc}
0.63 & 0 & 0 & 0 & 0.09 & 0 \\
0.07 & 0.43 & 0 & 0.024 & 0 & 0 \\
0.12 & 0 & 0.33 & 0 & 0 & 0.39 \\
0.12 & 0 & 0.32 & 0 & 0 & 0.38 \\
0.06 & 0 & 0.22 & 0.41 & 0.09 & 0
\end{array}\right)\left(\begin{array}{l}
\mu_{1} \\
\mu_{2} \\
\mu_{3} \\
\mu_{4} \\
\mu_{5} \\
\mu_{6}
\end{array}\right)=\left(\begin{array}{c}
1.00 \\
0.55 \\
0.61 \\
0.60 \\
0.56
\end{array}\right)
$$

Solving Equation (43), we obtained equation (44) below

$$
\left(\begin{array}{l}
\mu_{1} \\
\mu_{2} \\
\mu_{3} \\
\mu_{4} \\
\mu_{5} \\
\mu_{6}
\end{array}\right)=\left(\begin{array}{c}
11.82000 \\
11.92000 \\
11.85000 \\
11.75000 \\
9.82000 \\
12.23000
\end{array}\right)
$$

From equation (44), in order to serve patients with the space interval of 4 minutes. We have to divide the values in equation (44) by 4 . The recommended number of servers therefore for each node is given in equation (45)

$$
\left(\begin{array}{l}
\mu_{1} \\
\mu_{2} \\
\mu_{3} \\
\mu_{4} \\
\mu_{5} \\
\mu_{6}
\end{array}\right)=\left(\begin{array}{l}
2.95 \\
2.89 \\
2.96 \\
2.93 \\
2.45 \\
3.08
\end{array}\right) \approx\left(\begin{array}{l}
3 \\
3 \\
3 \\
3 \\
2 \\
3
\end{array}\right)
$$

The values in equation (45) indicates that in order for us to reduce the waiting time in the system to an acceptable level, node one will require a total of three servers, node two will require a total of three servers, node three will require a total of three servers, node four will require a total of three servers, node five will require a total of two servers and node six will require a total of three servers. With the new estimated number of servers at each node given by equation (45). The arrival rates for each node is assumed to remain the same since we do not have control over it. A new departure rates and the expected waiting time for each node is estimated. The new departure rates for nodes are as follows: 
$\mu_{1}=$ recommended departure rate per $4 \mathrm{mins}=\frac{3}{4}=0.6$ person per minute (46)

$\mu_{2}=$ recommended departwe rate per 4 mins $=\frac{3}{4}=0.6$ person per minute (47)

$\mu_{3}=$ recommended departure rate per 4 mins $=\frac{3}{4}=0.6$ person per minute (48)

$\mu_{4}=$ recommended departure rate per 4 mins $=\frac{3}{4}=0.6$ persons per minute(49)

$\mu_{5}=$ recommended departure rate per 4 mins $=\frac{2}{4}=0.5$ person per minute (50)

$\mu_{6}=$ recommended departure rate per 4 mins $=\frac{3}{4}=0.6$ person per minute (52)

Computations of New Expected Waiting Time in the System

For Node 1:

$\rho=\frac{\lambda_{1}}{\mu_{\perp}}=\frac{0.55}{0.6}=0.9$

The expected number of patients in the queue is

given as $l_{q}=\frac{\rho}{m-\rho}=\frac{0.9}{3-0.9}=0.4$ patients

The expected waiting time in the queue is given as

$w_{a i}=\frac{l_{9}}{A_{1}}=\frac{0.4}{0.65}=0.7$

The expected number of patients in the system is given as

$l_{s}=l_{q}+\rho=0.4+0.9$ Patients.

The expected waiting time of the patients in the system for node 1 is given as

$w_{1}=\frac{l_{s}}{\lambda_{1}}=\frac{0.4}{0.55}=2.4$ Minutes.

These parameters calculated for node 1 can be done for nodes $2-6$.

The expected waiting time in node 1 through node 6 were calculated as $\mathrm{w}_{1}=2.4$ minutes for node $1, \mathrm{w}_{2}=$ 3 minutes for node $2, w_{3}=1.8$ minutes, for node 3 , $\mathrm{W}_{4}=2.4$ minutes for node 4 ,

$\mathrm{w}_{5}=2.5$ minutes for node 5 and $\mathrm{w} 6=6.8$ minutes for node 6 .

Therefore, the total expected waiting time in the system after modification is given as

$W=W_{1}+W_{2}+W_{3}+W_{4}+W_{5}+W_{6}=$

$2.4+3+1.8+2.4+2.5+6.2=18.8 \approx 19$ minutes

These values are presented in Table 3
Table 3: Showing all the results obtained after modification

\begin{tabular}{lllllll}
\hline Nodes i & $\begin{array}{l}\text { Number of } \\
\text { Servers }\left(\mathrm{m}_{\mathrm{i}}\right)\end{array}$ & $\rho_{\mathrm{i}}$ & $\mathrm{Lq}$ & $\mathrm{Ls}$ & $\mathrm{W}_{\mathrm{q}}$ & $\mathrm{W}_{\mathrm{S}}$ \\
\hline 1 & 3 & 0.9 & 0.4 & 1.3 & 0.7 & 2.4 \\
2 & 3 & 1.7 & 1.3 & 3.0 & 1.3 & 3.0 \\
3 & 3 & 0.9 & 0.4 & 1.3 & 0.7 & 2.4 \\
4 & 3 & 1.0 & 0.5 & 1.5 & 0.8 & 2.5 \\
5 & 2 & 1.2 & 1.5 & 3.7 & 2.5 & 6.2 \\
6 & 3 & 0.9 & 0.4 & 1.3 & 0.7 & 2.3 \\
Total & 17 & 6.6 & 2.3 & 12.1 & 6.7 & 18.8 \\
\hline
\end{tabular}

It can be observed from the table that the total number of servers is 17 , this is the optimal number of servers that can reduce the waiting time to 18.8 minutes, approximately 19 minutes as presented on the table.

Table 4. Showing the comparison between current number of servers and optimal number of servers obtained

\begin{tabular}{lll}
\hline Nodes i & $\begin{array}{l}\text { Current number } \\
\text { of servers }\end{array}$ & $\begin{array}{l}\text { Optimal number } \\
\text { of servers obtained }\end{array}$ \\
\hline 1 & 4 & 3 \\
2 & 2 & 3 \\
3 & 1 & 3 \\
4 & 1 & 3 \\
5 & 1 & 2 \\
6 & 1 & 3 \\
Total & 10 & 17 \\
\hline
\end{tabular}

Conclusion: The network queuing system of Federal Polytechnic Bida school clinic has been effectively investigated and studied. The study has determined optimal number of servers at the nodes of the school clinic network queuing system to reduce waiting time of the patients. Results from the study is an important information to the management of Federal Polytechnic Bida school clinic for proper planning and efficient service delivery.

\section{REFERENCES}

Adaji, I (2018).A determination of optimal performance for the queuing system, an unpublished Master's thesis submitted to the Department of Mathematics, federal university of Technology, Minna, Niger State, Nigeria.

John, K.O (2010). Queuing Theory and Patient Satisfaction. An overview of Terminology and 
Application in Ante-natal Care Unit. Bulletin of Petroleum Gas University of Ploiesti. 61: 1-10.

Kandenmir, C; Cavas, I (2007).An application of queuing theory to the relationship between insulin level and number of insulin receptors. Turk. J. Biochem. 32: 32-38.

Kembe, M; Onah, M;lorkegh, E.S (2012). A Study of Waiting and Service cost of a Multi-Server Queuing Model in a Specialist Hospital. Inter. J. Sci. Tech. Res. 1 (8) 19-23.

Lakshmi, C; Sivakumar, A.I. (2013.). Application of queuing theory in health care: A literature review. Oper. Res. Health Care. 2: 25-39.
Lawal, A; Adaji, I; Abdulkadir, A (2019). A Study of Waiting and Service Cost of a Mult-Server Queuing System at National Health Insurance Scheme (NHIS) Unit of the General Hospital Minna. Transact Nig. Assoc. Math. Physics. 10:177-184

Olaniyi, (2004).Waiting for Orthopaedic surgery. Factors associated with waiting time and patient's opinion. Inter. J. Quality Healthcare.17: 133-140.

Schlechter (2009). Hershey Medical Center to open redesigned emergency room. The patriot News. 